\title{
Les présénilines et la voie de signalisation Notch
}

A ce jour, trois gènes impliqués dans les formes familiales précoces (à transmission autosomique dominante) de la maladie d'Alzheimer ont été identifiés [1] : les gènes presenilin 1 et 2 (PS1 et $P S 2)$ et le gène APP (amyloid precursor protein) [2]. Les gènes $P S 1$ et $P S 2$ codent pour des protéines transmembranaires localisées essentiellement dans la membrane du réticulum endoplasmique et dans les vésicules issues de l'appareil de Golgi. Plusieurs arguments s'accordent pour impliquer les présénilines dans le métabolisme de l'APP. En effet, les mutations dans ces gènes ont pour conséquence biologique l'accumulation du peptide neurotoxique $A \beta 42$, dérivé du précurseur de la protéine $\beta$-amyloïde (APP) et principal constituant des plaques séniles (m/s 1997, $n^{\circ} 1$, p. 106). La protéine transmembranaire APP subit deux coupures protéolytiques: une catalysée par la $\beta$-sécrétase, à l'extrémité amino-terminale de la séquence $\mathrm{A} \beta$ (figure 1), l'autre, catalysée par la $\gamma$-sécrétase, qui libère l'extrémité carboxy-terminale du peptide $A \beta$, permettant sa sécrétion. A ce jour, la nature moléculaire de ces deux sécrétases n'est pas connue. Par ailleurs, plusieurs observations suggèrent un rôle des présénilines dans la voie de signalisation Notch $\left(\mathrm{m} / \mathrm{s} 1997, n^{\circ} 8 / 9\right.$, p. 1036). Le gène sel-12, un homologue des gènes PS1 et PS2 chez le nématode, interagit avec le gène lin-12, un membre distant de la famille des récepteurs Notch. Une réduction de l'activité sel-12 supprime les effets associés à une augmentation de l'activité lin-12. En outre, les embryons de souris porteurs d'une mutation nulle dans le gène PS1 à l'état homozygote présentent des anomalies de développement semblables à celles qu'entraîne l'inactivation du gène Notch-1. Enfin, une co-localisation cellulaire des protéines Notch et présénilines, ainsi que des interactions protéiques directes, ont été observées dans des cellules de mammifères en culture et chez la drosophile.

Chez les vertébrés et les invertébrés, la famille des récepteurs transmembranaires Notch contrôle la différenciation de nombreux types cellulaires au cours du développement [3]. Au cours de leur transit vers la membrane plasmique, les protéines Notch subissent une première coupure protéolytique dans leur domaine extracellulaire, catalysée par une furine, (figure 1) [4] (m/s 1999, $n^{\circ} 3$, p. 414). Les deux fragments produits restent associés pour former un récepteur Notch fonctionnel. L'activation des récepteurs Notch entraîne une seconde coupure protéolytique libérant un fragment intracellulaire (NICD, Notch intracellular domain) qui migre dans le noyau où il participe à la formation d'un complexe activateur de la transcription.

De cette similitude certaine dans la série de coupures protéolytiques que subissent Notch et l'APP est née l'idée que les présénilines pourraient être impliquées, directement ou indirectement, dans les protéolyses successives des récepteurs Notch, rôle que plusieurs travaux récents tentent d'établir [5-8]. Chez la drosophile, la perte de l'activité du gène presenilin et la perte de fonction du gène Notch entrainent des anomalies de développement semblables $[6,8]$. Cependant, ni la production ni la localisation subcellulaire de la protéine Notch ne sont affectées $[6,8]$ et la première étape de clivage subie par le récepteur Notch se produit normalement $[5,8]$. Les défauts phénotypiques observés dans les mutants pre- senilin-/- résultent en fait d'une absence de la transmission du signal induit par l'activation de Notch, comme le confirme l'absence d'activité nucléaire Notch [6]. Les présénilines seraient impliquées dans la libération du fragment intracellulaire (NICD) de la membrane plasmique : ainsi, si on exprime dans des neurones $\mathrm{PS1}^{-/-}$une forme constitutivement activée du récepteur Notch-1 de souris $(\mathrm{N} \Delta \mathrm{E})$ (figure 1), la quantité de NICD produite est très fortement réduite comparée à celle détectée après expression de cette même construction dans des neurones sauvages [5]. Or, cette forme $\mathrm{N} \Delta \mathrm{E}$ tronquée code uniquement pour les domaines intracellulaire et transmembranaire de Notch-1. La libération du fragment NICD de $\mathrm{N} \Delta \mathrm{E}$ implique une seule coupure protéolytique dans son domaine sous-membranaire. Second argument, l'enzyme responsable de la libération de NICD présente les mêmes propriétés pharmacologiques que la $\gamma$-sécrétase. Elle est notamment sensible à un inhibiteur spécifique de l'activité $\gamma$-sécrétase [5]. Des résultats semblables mais controversés (voir ci-dessous), ont été obtenus chez la drosophile [6]. Les auteurs utilisent une stratégie fondée sur la construction d'un récepteur Notch constitutivement activé ( $\mathrm{N} \Delta \mathrm{E})$ auquel est couplé, dans sa partie intracellulaire, une activité biologique strictement nucléaire (activité de liaison à l'ADN) qui peut être détectée in vivo après transgenèse. La détection de l'activité nucléaire de Notch permet de révéler de manière indirecte la présence de NICD dans le noyau, témoin de sa libération de la membrane. Dans un contexte où le gène presenilin est muté, aucune activité nucléaire Notch n'est détectée [6].

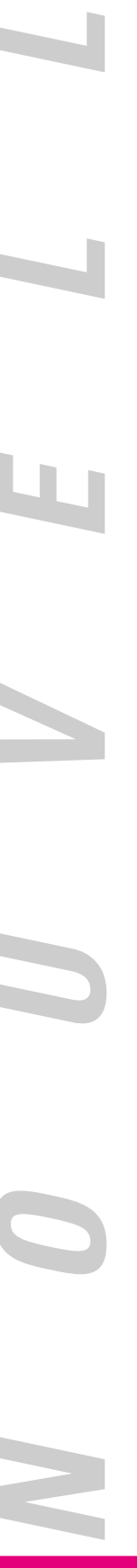




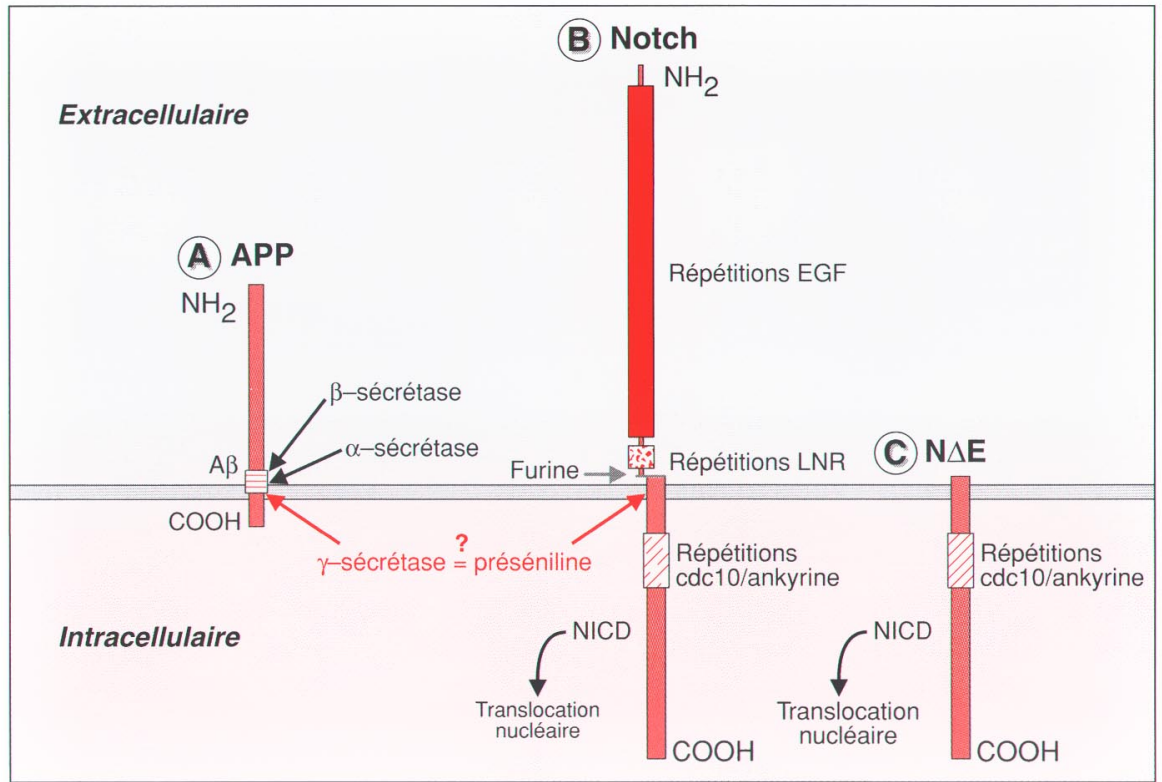

Figure 1. Cascade protéolytique impliquée dans la maturation des protéines APP et Notch. A. L'APP subit une première étape de coupure protéolytique, catalysée par l' $\alpha$ - ou la $\beta$-sécrétase, au niveau de l'un des deux sites extracellulaires. À la suite de ce clivage extracellulaire, une seconde coupure, catalysée par la $\gamma$-sécrétase, se produit à l'extrémité carboxy-terminale de la séquence $A \beta$. Le clivage de I'APP par la $\beta$-sécrétase puis par la $\gamma$-sécrétase permet la sécrétion du peptide $A \beta$. B. Notch subit une première coupure protéolytique, catalysée par une furine, dans son domaine extracellulaire au cours de son transit vers la membrane plasmique. Les deux fragments résultants restent associés à la membrane pour former un récepteur Notch fonctionnel. En réponse à la fixation de son ligand extracellulaire, le récepteur Notch activé subit une seconde coupure protéolytique (dans son domaine intracellulaire) libérant un fragment NICD capable de transloquer dans le noyau et d'activer la transcription de gènes. Trois types de répétitions sont présentes dans la protéine Notch : EGF (epidermal growth factor), Lin12/Notch (LNR) et ankyrines. C. Représentation de la construction constitutivement activée du récepteur Notch (NDE) utilisée dans les expériences rapportées dans ce texte.

Les deux groupes concluent donc à la nécessité des présénilines pour la libération de NICD de la membrane plasmique. Les présénilines agiraient en facilitant l'activité des protéases responsables de la libération de NICD, à moins qu'elles ne soient elles-mêmes des protéases. Cette dernière hypothèse est aussi celle d'un autre groupe qui démontre que la mutation de deux acides aspartiques conservés dans la protéine PS1 abolit le clivage de l'APP par l'activité $\gamma$-sécrétase [7].

Cependant, un rôle des présénilines dans le transport des protéines a aussi été suggéré, et la localisation subcellulaire de ces protéines plaide

effet, la protéine SPE-4, un autre homologue de la protéine PS1 chez le nématode, a une action de stabilisation des vésicules de l'appareil de Golgi, et intervient dans le transport et le recyclage des protéines au cours de la spermatogenèse.

Contrairement aux résultats mentionnés ci-dessus, une étude réalisée chez la drosophile presenilin montre toutefois qu'une construction Notch membranaire constitutivement activée (semblables à $\mathrm{N} \Delta \mathrm{E}$ ) possède une activité de signalisation chez les mutants presenilin. En effet, l'expression de la construction $\mathrm{N} \Delta \mathrm{E}$ bloque la différenciation des cellules nerveuses de manière égale dans un contexte sauvage ou dans un contexte mutant pour le gène presenilin [8]. L'activité presenilin est donc requise à une étape en aval de la coupure libérant NICD de la membrane, coupure qui se produit de manière constitutive dans les constructions $\mathrm{N} \Delta \mathrm{E}$. Les présénilines pourraient donc être impliquées dans le ciblage et le transport intracellulaire des substrats ou des protéases impliquées dans les clivages de type $\gamma$-sécrétase.

L'importance des présénilines dans la voie de signalisation Notch est maintenant bien établie. Leur rôle précis reste cependant mal déterminé. Ces résultats doivent aussi rendre prudents dans l'utilisation d'inhibiteurs spécifiques de l'activité $\gamma$-sécrétase pour traiter les patients atteints de la maladie d'Alzheimer. En effet, l'utilisation de tels produits pourrait également inactiver la voie de signalisation Notch et entraîner une immunodéficience du fait du rôle important de la protéine Notch-1 dans l'hématopoïèse tout au long de la vie.

\section{Magalie Lecourtois}

Division of Mammalian Development, National Institute for Medical Research, The Ridgeway, Mill Hill, Londres, NW7 1AA, Royaume-Uni.

1. Octave JN, Macq AM, Philippe B. Le précurseur du peptide amyloïde de la maladie d'Alzheimer. Med Sci $1995 ; 11$ : 1251-9.

2. Selkoe DJ. Translating cell biology into therapeutic advances in Alzheimer's disease. Nature 1999 ; 399 (suppl) : A23-31.

3. Schweisguth F, Israel A. Signalisation intercellulaire par le récepteur Notch: conservation de la drosophile aux mammifères. Med Sci 1996; 12 : 155-163 4. Chan YM, Jan YN. Roles for proteolysis and trafficking in notch maturation and signal transduction. Cell 1998; 94 : 423-6.

5. De Strooper B, et al. A presenilin-1 dependent gamma-secretase-like protease mediates release of Notch intracellular domain. Nature 1999; 398 : 518-22.

6. Struhl G, Greenwald I. Presenilin is required for activity and nuclear access of Notch in Drosophila. Nature 1999; 398: 522-5.

7. Wolfe MS, et al. Two transmembrane aspartates in presenilin-1 required for presenilin endoproteolysis and gamma-secretase activity. Nature 1999 ; 398: 513-7.

8. Ye Y, Lukinova N, Fortini, ME. Neurogenic phenotypes and altered Notch processing in Drosophila Presenilin mutants. Nature 1999; 398 : 525-9. 